\title{
Reflection from a Flat Dielectric Film with Negative Refractive Index
}

\author{
Pierre Hillion \\ Institut Henri Poincaré, 86 Bis Route de Croissy, 78110, Le Vésinet, France
}

Received on 20 July, 2007

\begin{abstract}
We analyse the reflection of a TM electromagnetic field first on a flat dielectric film and second on a Veselago film with negative refractive index, both films being deposited on a metallic substrat acting as a mirror. An incident harmonic plane wave generates inside a conventional dielectric film a refracted propagating wave and an evanescent wave that does not contribute to reflection on the metallic substrat so that part of the information conveyed by the incident field is lost. At the opposite, inside a Veselago film, evanescent waves are changed into outbursting waves reflecting on the metallic substrat and participating to the total reflected field from the metallic film without loss if information.
\end{abstract}

Keywords: Dielectric Film; Negative Refractive Index; Veselago film

\section{INTRODUCTION}

The properties of a dielectric film located between two homogeneous media is of particular interest in optics, for instance dielectric films are commonly used to reduce or eliminate unwanted reflections. These properties depend strongly on the refractive index profile [1] of the dielectric material but till now the refractive index was always assumed positive.

But some years ago, Veselago [2] analysed what would happen to electromagnetic waves immersed in a hypothetical medium with negative permittivity and permeability. In the mid 1990's people began looking into the possibility to engineering materials with these exotic properties in some frequency range and practical realizations were proposed in 1993 [3] while a first experiment was performed in 2001 [4]. Since then, works on this fascinating subject are flourishing with more than 200 papers in 2003 and still more expected in years to come [5].

The refractive index in Veselago materials is negative implying that the phase of a wave decreases rather than advances along propagation through these media leading to important implications for nearly all electromagnetic phenomena as pointed out by Veselago himself. So, a natural question is what happens when a Veselago film takes the place of a conventional dielectric film deposited on a metallic substrat.

To investigate this situation, we consider a TM electromagnetic field impinging from the half-space $z>0$ on a flat coated mirror made of a Veselago film with negative permittivity, permeability, refractive index and thickness $d$ deposited on a mirror located at $z=-d$ and we look for the TM field reflected by this coated mirror. For sake of comparison, we first analyse the same problem with a conventional dielectric film as coating.

\section{TM FIELD IMPINGING ON A DIELECTRIC FILM}

For a TM field, Maxwell's equations with $\exp (i \omega t)$ implicit reduce in a dielectric of permittivity $\varepsilon$ and permeability $\mu$ to

$$
\partial_{x} H_{y}=i \omega \varepsilon / c E_{z}, \quad \partial_{z} E_{x}-\partial_{x} E_{z}=-i \omega \mu / c H_{y}
$$

and $H_{y}$ is solution of the 2D-Helmholtz equation

$$
\left(\partial_{x}^{2}+\partial_{z}^{2}+\omega^{2} n^{2} / c^{2}\right) H_{y}=0, n^{2}=\varepsilon \mu .
$$

Changing $\varepsilon, \mu, n$ into $\varepsilon_{0}, \mu_{0}, n_{0}$ in Eqs.(1), (1a) gives the corresponding equations for the components $E_{x}{ }^{e}, E_{z}{ }^{e}, H_{y}{ }^{e}$ of the TM field in the half space $z>0$.

\section{A. Impedance boundary conditions}

To get the TM reflected field, we need the boundary conditions on the dielectric film and using the Idemen technique [6] we introduce the Fourier transform in which $n$ is the refractive index,

$$
\left\{e_{x}, e_{z}, h_{y}\right\}(\xi, z)=\int_{-\infty}^{\infty} d x \exp (i n \xi x)\left\{E_{x}, E_{z}, H_{y}\right\}(x, z)
$$

and similarly for $e_{x}^{e}, e_{z}^{e}, h_{y}^{e}$.

The equations (1) and (1a) become

$$
\begin{gathered}
\partial_{z} H_{y}=-i \omega \varepsilon / c e_{x}, \\
n \xi h_{y}=\omega \varepsilon / c e_{z}, \quad \partial_{z} e_{x}-i n \xi e_{z}=-i \omega \mu / c h_{y}, \\
\left(\partial_{z}^{2}+\lambda^{2}\right) h_{y}=0, \lambda^{2}=n^{2}\left(\omega^{2} / c^{2}-\xi^{2}\right) .
\end{gathered}
$$

The TM field inside the dielectric is the sum of a wave propagating in the $z<0$ direction and of the wave reflected in the $z>0$ direction by the $z=-d$ mirror. Then, since these waves are solutions of Eqs. (3), (3a) we may write $h_{y}(\xi, z)$. For $|\xi| \leq \omega / c$,

$$
h_{y}(\xi, z)=A(\xi)[\exp (i \lambda z)+r(\xi) \exp (-i \lambda z)]
$$

$$
\partial_{z} H_{y}=-i \omega \varepsilon / c E_{x}
$$




$$
\lambda=n\left(\omega^{2} / c^{2}-\xi^{2}\right)^{1 / 2}
$$

in which $A(\xi)$ is an arbitrary amplitude and $r(\xi)$ the reflection coefficient at $z=-d$. For $|\xi|>\omega / c$,

$$
\begin{gathered}
h_{y}(x, z)=A^{\dagger}(\xi)\left[\exp \left(\lambda^{\dagger} z\right)+r^{\dagger}(\xi) \exp \left(-\lambda^{\dagger} z\right)\right] \\
\lambda^{\dagger}=n\left(\xi^{2}-\omega^{2} / c^{2}\right)^{1 / 2}
\end{gathered}
$$

Since $z<0$, the first term in (4a) is an evanescent wave unable to reach the $z=-d$ mirror so that $r^{\dagger}(\xi)=0$ implying that evanescent waves do not contribute to the TM field inside the dielectric and consequently no more to $h_{y}^{e}(\xi, z)$ outside the film.
Substituting (4) into (3) gives $e_{z}=c n \xi / \omega e h_{y}$ and

$$
e_{x}(x, z)=-\lambda c / \omega \varepsilon A(\xi)[\exp (i \lambda z)-r(\xi) \exp (-i \lambda z)]
$$

for $|\xi| \leq \omega c$. The continuity of $e_{x}, h_{y}$ at the $z=0$ face on which impinges the TM field implies the boundary conditions

$$
\left[e_{x}-e_{x}^{e}\right]_{z=0}=0,\left[h_{y}-h_{y}^{e}\right]_{z=0}=0 .
$$

Substituting (4) and (5) into (6) gives

$$
A(\xi)[1+r(\xi)]=h_{y}^{e}(\xi, 0), A(\xi)[1-r(\xi)]=-\omega \varepsilon / c \lambda e_{x}^{e}(\xi, 0),
$$

from which we get, since $\partial_{z} h_{y}^{e}=-i \omega \varepsilon_{0} / c e_{x}^{e}$,

$$
\begin{gathered}
2 A(\xi)=h_{y}^{e}(\xi, 0)-\omega \varepsilon / c \lambda e_{x}^{e}(x, 0)=h_{y}^{e}(\xi, 0)+i \varepsilon / \lambda \varepsilon_{0}\left[\partial_{z} h_{y}^{e}(\xi, z)\right]_{z=0}, \\
2 A(\xi) r(\xi)=h_{y}^{e}(\xi, 0)+\omega \varepsilon / c \lambda e_{x}^{e}(\xi, 0)=h_{y}^{e}(\xi, 0)-i \varepsilon / \lambda \varepsilon_{0}\left[\partial_{z} h_{y}^{e}(\xi, z)\right]_{z=0} .
\end{gathered}
$$

Now $\left[e_{x}(\xi, z)\right]_{z=-d}=0$ on the $z=-d$ mirror which implies according to (5)

$$
\exp (-i \lambda d)-r(\xi) \exp (i \lambda d)=0 .
$$

Substituting (8) into (9) gives the impedance boundary condition satisfied by $h_{y}^{e}(\xi, z)$ on the $z=0$ face of the dielectric film with $\partial_{z} h_{y}^{e}(\xi, 0)=\delta_{z}\left[h_{y}^{e}(\xi, 0)\right]_{z=0}$,

$$
\cos (\lambda d) \partial_{z} h_{y}^{e}(\xi, 0)-\lambda \varepsilon_{0} / \varepsilon \sin (\lambda d) h_{y}^{e}(\xi, 0)=0,
$$

that we write

$$
\begin{gathered}
\partial_{z} h_{y}^{e}(\xi, 0)-a(\xi) h_{y}^{e}(\xi, 0)=0, \\
a(\xi)=\varepsilon_{0} / \varepsilon \lambda(\xi) \tan [\lambda(\xi) d]
\end{gathered}
$$

with

$$
\lambda(\xi)=n\left(\omega^{2} / c^{2}-\xi^{2}\right)^{1 / 2}
$$

\section{B. TM reflected field on the flat dielectric film}

The TM field with the component $H_{y}{ }^{e}(x, z)$ in the half space $z>0$ above the dielectric film is the sum of an incident and reflected field with respective components $H_{y}{ }^{i}(x, z)$ and $H_{y}^{r}(x, z)$,

$$
H_{y}^{e}(x, z)=H_{y}^{i}(x, z)+H_{y}^{r}(x, z),
$$

with the Fourier transform

$$
h_{y}^{e}(\xi, z)=h_{y}^{i}(\xi, z)+h_{y}^{r}(\xi, z) .
$$

Taking into account (12a) we may write (11),

$$
\begin{gathered}
\partial_{z} h_{y}^{r}(\xi, 0)-a(\xi) h_{y}^{r}(\xi, 0)=-f(\xi) \\
f(\xi)=\partial_{z} h_{y}^{i}(\xi, 0)-a(\xi) h_{y}^{i}(\xi, 0) .
\end{gathered}
$$

Assuming specular the reflection of the TM incident field on the flat impedance film gives

$$
h_{y}^{r}(\xi, z)=R(\xi) h_{y}^{i}(\xi,-z)
$$

in which $R(\xi)$ is the reflection coefficient of the impedance film and substituting (14) into (13), we get

$$
\begin{gathered}
R(\xi)=f(\xi) / g(\xi), \\
g(\xi)=\partial_{z} h_{y}^{i}(\xi, 0)+a(\xi) h_{y}^{i}(\xi, 0),|\xi| \leq \omega / c .
\end{gathered}
$$

Since evanescent waves do not contribute to reflection, the inverse Fourier transform of $h_{y}^{r}(\xi, z)$,

$$
H_{y}^{r}(x, z)=\left(\frac{n}{2 \pi}\right) \int_{-\infty}^{\infty} d \xi \exp (-i n \xi x) R(\xi) h_{y}^{i}(\xi,-z)
$$

reduces to

$$
H_{y}^{r}(x, z)=\left(\frac{n}{2 \pi}\right) \int_{-\omega / c}^{\omega / c} d \xi \exp (-i n \xi x) \frac{f(\xi)}{g(\xi)} h_{y}^{i}(\xi,-z) .
$$

and since the Fourier transform $e_{x}^{i}(\xi, z), e_{z}^{i}(\xi, z), h_{y}^{i}(\xi, z)$ of the incident field is solution of Eq.(3) with $\varepsilon, \mu$ changed into $\varepsilon_{0}, \mu_{0}$, we get from (3) and (17) 


$$
\begin{aligned}
& E_{x}^{r}(x, z)=\left(i n c / 2 \pi \varepsilon_{0} \omega\right) \int_{-\omega / c}^{\omega / c} d \xi \exp (-i n \xi x) f(\xi) / g(\xi) \partial_{z} h_{y}^{i}(\xi,-z), \\
& E_{z}^{r}(x, z)=\left(n 2 c / 2 \pi \varepsilon_{0} \omega\right) \int_{-\omega / c}^{\omega / c} d \xi \exp (-i n \xi x) \xi f(\xi) / g(\xi) h_{y}^{i}(\xi,-z),
\end{aligned}
$$

which determines the TM field reflected by the flat impedance film.

\section{Harmonic plane wave reflection}

Because of the importance, to be discussed in Sec.4, of harmonic plane waves as building blocks of electromagnetic beams, we consider as incident field a TM harmonic plane wave with the $H_{y}$-component,

$$
H_{y}^{i}(x, z)=A \exp \left[i \xi n_{0} / c(x \sin \theta+z \cos \theta)\right], n_{0}=\left(e_{0} m_{0}\right)^{1 / 2},
$$

where $A$ is a constant amplitude. The Fourier transform of (18) is

$$
h_{y}^{i}(\xi, z)=2 \pi A \exp \left[-i k_{0} z \cot \theta\right) \delta\left(n \xi-k_{0}\right), k_{0}=-\omega n_{0} / c \sin \theta
$$

in which $\delta$ is the Dirac distribution.

Taking into account (18a) and changing $z$ into $-z$, the Fourier transform (17) becomes

$$
H_{y}^{r}(x, z)=n A \exp \left(i k_{0} z \cot \theta\right) \int_{-\omega / c}^{\omega / c} d \xi \exp (-i n \xi x) f(\xi) / g(\xi) \delta\left(n \xi-k_{0}\right)
$$

null for $k_{0} / n$ outside $(-\omega / c, \omega / c)$ while for $k_{0} / n$ in this interval

$$
H_{y}^{r}(x, z)=A f\left(k_{0} / n\right) / g\left(k_{0} / n\right) \exp \left[i \omega n_{0} / c(x \sin \theta-z \cos \theta)\right],
$$

in which we get from (13), (15) and (18a)

$$
f\left(k_{0} / n\right) / g\left(k_{0} / n\right)=\left[i k_{0} \cot \theta-a\left(k_{0} / n\right)\right]\left[i k_{0} \cot \theta+a\left(k_{0} / n\right)\right]^{-1},
$$

while according to (11) and (11a)

$$
a\left(k_{0} / n\right)=\varepsilon_{0} / \varepsilon \lambda\left(k_{0} / n\right) \tan \left[\lambda\left(k_{0} / n\right) d\right], \lambda\left(k_{0} / n\right)=\omega n c^{-1}\left(1-n_{0}^{2} \sin ^{2} \theta / n^{2}\right)^{1 / 2} .
$$

Finally, taking into account (19) and definition (18a) of $k_{0}$, we get from (17a)

$$
E_{x}^{r}(x, z)=-\cos \theta H_{y}^{r}(x, z), E_{z}^{r}(x, z)=+\sin \theta H_{y}^{r}(x, z)
$$

The relations (19) and (21) represent the specularly reflected TM field from a flat dielectric film for an incident harmonic plane wave. But, since evanescent waves do not reach the $z=-d$ mirror, the information conveyed by the incident field for $\xi>|\omega / c|$ is lost for the reflected field.

\section{TM FIELD IMPINGING ON A VESELAGO FILM}

Permittivity, permeability, refractive index in Veselago materials are negative but to investigate the reflection of a TM field on a Veselago film it is easier to perform on the expressions obtained in Sec. 2 the following transpositions in which $\varepsilon_{1}, \mu_{1}, n_{1}, \lambda_{1}, \lambda_{1}^{\dagger}$ are positive,

$$
\begin{gathered}
\varepsilon, \mu, n, \lambda, \lambda^{\dagger} \Rightarrow-\left(\varepsilon_{1}, \mu_{1}, n_{1}, \lambda_{1}, \lambda_{1}^{\dagger}\right), \\
\lambda_{1}=n_{1}\left(\omega^{2} / c^{2}-\xi^{2}\right)^{1 / 2}, \lambda_{1}^{\dagger}=n_{1}\left(\xi^{2}-\omega^{2} / c^{2}\right)^{1 / 2} .
\end{gathered}
$$

Then, the Fourier transform (2) being defined with the exponential $\exp \left(-i n_{1} \xi x\right)$, the equations (3), (3a) become

$$
\begin{gathered}
\partial_{z} h_{y}=i \omega \varepsilon_{1} / c e_{x}, n_{1} \xi h_{y}=\omega \varepsilon_{1} / c e_{z} \\
\partial_{z} e_{x}+i n_{1} \xi_{e_{z}}=i \omega \mu_{1} / c h_{y}
\end{gathered}
$$




$$
\left(\partial_{z}^{2}+\lambda_{1}^{2}\right) h_{y}=0, \lambda_{1}^{2}=n_{1}\left(\omega^{2} / c^{2}-\xi^{2}\right) .
$$

Applying the transpositions (22) to Eqs. (4), (4a) gives

$$
\begin{gathered}
h_{y}(x, z)=A_{1}(\xi)\left[\exp \left(-i \lambda_{1} z\right)+r_{1}(\xi) \exp \left(i \lambda_{1} z\right)\right] \\
\lambda_{1}=n_{1}\left(\omega^{2} / c^{2}-\xi^{2}\right)^{1 / 2} \\
h_{y}(x, z)=A_{1}^{\dagger}(\xi)\left[\exp \left(-\lambda_{1}^{\dagger} z\right)+r_{1}^{\dagger}(\xi) \exp \left(\lambda_{1}^{\dagger} z\right)\right], \\
\lambda_{1}^{\dagger}=n_{1}\left(\xi^{2}-\omega^{2} / c^{2}\right)^{1 / 2},
\end{gathered}
$$

with a label different from (4) and (4a) for amplitudes and reflection coefficients. We first note from (24a) that the phase velocity in Veselago media is antiparallel to the Poynting vector but the main changes come from (24b). For $z<0$, the first exponential term in (24b) corresponds not to an evanescent wave but to an outbursting wave whose amplitude increases with the distance (on a distance limited by the breakdown of the theory but supposed greater than the film thickness). This outbursting wave gives birth on the $z=-d$ mirror to a reflected field attenuated with the distance according to the second exponential term in (24b). We also assume this attenuation not strong enough to make negligible the reflected field at the output of the Veselago film and in fact, for a reflection coefficient of modulus unity on the mirror, the reflected field has the same amplitude as the incident field. Consequently, we have to take into account reflections for $|\xi|>\omega / c$.

Then using (23), we get from (24) and (24a) for the $e_{x}$ component

$$
\begin{gathered}
e_{x}(\xi, z)=-\lambda_{1} c / \omega \varepsilon_{1} A_{1}(\xi)\left[\exp \left(-i \lambda_{1} z\right)+r_{1}(\xi) \exp \left(i \lambda_{1} z\right)\right], \quad|\xi| \leq \omega / c, \\
e_{x}(x, z)=-i \lambda_{1}^{\dagger} c / \omega \varepsilon_{1} A_{1}^{\dagger}(\xi)\left[\exp \left(-\lambda_{1}^{\dagger} z\right)+r_{1}^{\dagger}(\xi) \exp \left(i \lambda_{1}^{\dagger} z\right)\right],|\xi|>\omega / c
\end{gathered}
$$

and we shall consider separately the contributions to the reflected TM field of the harmonic waves (24a), (25a) and of the outbursting waves (24b), (25b).

\section{A. Harmonic waves: $\lambda_{1}=n_{1}\left(\omega^{2} / c^{2}-\xi^{2}\right)^{1 / 2}$}

We have just to apply the transpositions (22) to the expression obtained in Sec. 2. The impedance boundary condition (11) becomes

$$
\begin{gathered}
\partial_{z} h_{y}^{e}(\xi, 0)-a_{1}(\xi) h_{y}^{e}(\xi, 0)=0, \\
a_{1}(\xi)=-\varepsilon_{0} / \varepsilon_{1} \lambda_{1}(\xi) \tan \left[\lambda_{1}(\xi) d\right],
\end{gathered}
$$

while we get from (13), (14), (15)

$$
\partial_{z} h_{y}^{r}(\xi, 0)-a_{1}(\xi) h_{y}^{r}(\xi, 0)=-f_{1}(\xi),
$$

$$
f_{1}(\xi)=\partial_{z} h_{y}^{i}(\xi, 0)-a_{1}(\xi) h_{y}^{i}(\xi, 0)
$$

$$
R_{1}(\xi)=f_{1}(\xi) / g_{1}(\xi)
$$

$$
g_{1}(\xi)=\partial_{z} h_{y}^{i}(\xi, 0)+a_{1}(\xi) h_{y}^{i}(\xi, 0)
$$

So finally according to (17)

$$
H_{y}^{r}(x, z)=-\left(n_{1} / 2 \pi\right) \int_{-\omega / c}^{\omega / c} d \xi \exp \left(-i n_{1} \xi x\right) f_{1}(\xi) / g_{1}(\xi) h_{y}^{i}(\xi,-z) .
$$

Taking into account (1) with $\varepsilon_{0}, \mu_{0}$, permittivity and permeability in the half space $z>0$,

$$
\begin{aligned}
& E_{x}^{r}(x, z)=-\left(i n_{1} c / 2 \pi \omega \varepsilon_{0}\right) \int_{-\omega / c}^{\omega / c} d \xi \exp \left(-i n_{1} \xi x\right) f_{1}(\xi) / g_{1}(\xi) \partial_{z} h_{y}^{i}(\xi,-z), \\
& E_{z}^{r}(x, z)=\left(n_{1} 2 c / 2 \pi \omega \varepsilon_{0}\right) \int_{-\omega / c}^{\omega / c} d \xi \exp \left(-i n_{1} \xi x\right) x f_{1}(\xi) / g_{1}(\xi) h_{y}^{i}(\xi,-z) .
\end{aligned}
$$

Suppose now $n_{1}=n$, then according to (11) and (26) $a_{1}(\xi)=-a(\xi)$ changing $f_{1}(\xi) / g_{1}(\xi)$ into $g(\xi) / f(\xi)$ which 
is an inversion of the reflection coefficient. This suggests that a twinned set made of a dielectric and of a Veselago films with the same thickness and $n=n_{1}$, deposited on a mirror behaves as a perfect mirror at the frequencies for which (17) and (29) are valid.

B. Outsbursting waves: $\lambda_{1}^{\dagger}=n_{1}\left(\xi^{2}-\omega^{2} n_{1}^{2} / c^{2}\right)^{1 / 2}$

Since (24b), (25b) are deduced from (24a), (25a) by changing $\lambda_{1}$ into $-i \lambda_{1}^{\dagger}$, the results of Sec. 3.1 are at once available and with this substitution the boundary impedance condition (26) becomes

$$
\begin{gathered}
\partial_{z} h_{y}^{e}(\xi, 0)-a_{1}^{\dagger}(\xi) h_{y}^{e}(\xi, 0)=0 \\
a_{1}^{\dagger}(\xi)=\varepsilon_{0} / \varepsilon_{1} \lambda_{1}^{\dagger}(\xi) \tanh \left[\lambda_{1}^{\dagger}(\xi) d\right]
\end{gathered}
$$

and we get from (27), (28)

$$
\begin{gathered}
\partial_{z} h_{y}^{r}(\xi, 0)-a_{1}^{\dagger}(\xi) h_{y}^{r}(\xi, 0)=-f_{1}^{\dagger}(\xi) \\
f_{1}^{\dagger}(\xi)=\partial_{z} h_{y}^{i}(\xi, 0)-a_{1}^{\dagger}(\xi) h_{y}^{i}(\xi, 0)
\end{gathered}
$$

$$
R_{1}^{\dagger}(\xi)=f_{1}^{\dagger}(\xi) / g_{1}^{\dagger}(\xi)
$$

$$
g_{1}^{\dagger}(\xi)=\partial_{z} h_{y}^{i}(\xi, 0)+a_{1}^{\dagger}(\xi) h_{y}^{i}(\xi, 0)
$$

Now, since $|\xi|>\omega / c$, the inverse Fourier transform (16) of $h_{y}^{r}(\xi, z)=R_{1}^{\dagger}(x) h_{y}^{i}(\xi,-z)$ is

$$
\begin{gathered}
H_{y}^{r}(x, z)=\left(n_{1} / 2 \pi\right)\left(\int_{\infty}^{\alpha}+\int_{\beta}^{\infty}\right) d \xi \exp \left(\operatorname{in}_{1} \xi x\right) f_{1}^{\dagger}(\xi) / g_{1}^{\dagger}(\xi) h_{y}^{i}(\xi,-z) \\
\alpha=-\omega n_{1} / c \quad, \quad \beta=\omega n_{1} / c
\end{gathered}
$$

Still using (1) with $\varepsilon_{0}, \mu_{0}$,

$$
\begin{aligned}
& E_{x}^{r}(x, z)=\left(\operatorname{in}_{1} c / 2 \pi \varepsilon_{0} \omega\right)\left(\int_{\infty}^{\alpha}+\int_{\beta}^{\infty}\right) d \xi \exp \left(i n_{1} \xi x\right) f_{1}^{\dagger}(\xi) / g_{1}^{\dagger}(\xi) \partial_{z} h_{y}^{i}(\xi,-z), \\
& E_{z}^{r}(x, z)=\left(n_{1} 2 c / 2 \pi \varepsilon_{0} \omega\right)\left(\int_{\infty}^{\alpha}+\int_{\beta}^{\infty}\right) d \xi \exp \left(i n_{1} \xi x\right) \xi f_{1}^{\dagger}(\xi) / g_{1}^{\dagger}(\xi) h_{y}^{i}(\xi,-z),
\end{aligned}
$$

which is the contribution of outbursting waves to the TM field reflected from a flat Veselago film. So, at the difference of what happens with a dielectric film, all the information conveyed by the incident field is present with some distortion in the reflected field.

\section{Harmonic plane wave reflection}

We now suppose that the harmonic plane wave (18) with the Fourier transform (18a) impinges on the flat Veselago film.
For $|\xi| \leq \omega / c$, applying the substitutions (22) to (20) and (20a) gives at once,

$H_{y}{ }^{r}(x, z)=A f_{1}\left(k_{0} / n_{1}\right) / g_{1}\left(k_{0} / n_{1}\right) \exp \left[i \omega n_{0} / c(x \sin \theta-z \cos \theta)\right]$

$$
E_{x}^{r}(x, z)=\cos \theta H_{y}^{r}(x, z), E_{z}^{r}(x, z)=\sin \theta H_{y}^{r}(x, z) .
$$

According to (20) and (20a),

$$
f_{1}\left(k_{0} / n_{1}\right) / g_{1}\left(k_{0} / n_{1}\right)=\left[i k_{0} \cot \theta-a_{1}\left(k_{0} / n_{1}\right)\right]\left[i k_{0} \cot \theta+a_{1}\left(k_{0} / n_{1}\right)\right]^{-1}
$$

in which

$$
a_{1}\left(k_{0} / n_{1}\right)=\left(\varepsilon_{0} / \varepsilon_{1}\right) \lambda_{1}\left(k_{0} / n_{1}\right) \tan \left[\lambda_{1}\left(k_{0} / n_{1}\right) d\right], \lambda_{1}\left(k_{0} / n_{1}\right)=\omega n_{1} c^{-1}\left(1-n_{0}^{2} \sin ^{2} \theta / n_{1}^{2}\right)^{1 / 2} .
$$


For $\xi>\omega / c$, since according to (18a)

$$
\begin{gathered}
h_{y}^{i}(\xi,-z)=2 \pi A \exp \left(i k_{0} z \cot \theta\right) \delta\left(n x-k_{0}\right), k_{0}=-\omega n_{0} / c \sin \theta \\
H_{y}^{r}(x, z)=n_{1} A \exp \left(i k_{0} z \cot \theta\right) \int_{\omega / c}^{\infty} d \xi \exp \left(i n_{1} \xi x\right) f_{1}^{\dagger}(\xi) / g_{1}^{\dagger}(\xi) \delta\left(n_{1} x-k_{0}\right),
\end{gathered}
$$

giving for $k_{0} / n_{1}<\omega / c$

$$
H_{y}^{r}(x, z)=A f_{1}^{\dagger}\left(k_{0} / n_{1}\right) / g_{1}^{\dagger}\left(k_{0} / n_{1}\right) \exp \left[i \omega n_{0} / c(x \sin \theta-z \cos \theta)\right]
$$

in which

$$
f_{1}^{\dagger}\left(k_{0} / n_{1}\right) / g_{1}^{\dagger}\left(k_{0} / n_{1}\right)=\left[i k_{0} \cot \theta-a_{1}^{\dagger}\left(k_{0} / n_{1}\right)\right]\left[i k_{0} \cot \theta+a_{1}^{\dagger}\left(k_{0} / n_{1}\right)\right]^{-1}
$$

with

$$
a_{1}^{\dagger}\left(k_{0} / n_{1}\right)=\varepsilon_{0} / \varepsilon_{1} \lambda_{1}^{\dagger}\left(k_{0} / n_{1}\right) \tanh \left[\lambda_{1}^{\dagger}\left(k_{0} / n_{1}\right) d\right], \lambda_{1} \dagger\left(k_{0}\right)=\omega n_{1} c^{-1}\left(n_{0}^{2} \sin ^{2} \theta / n_{1}^{2}-1\right)^{1 / 2}
$$

Substituting (38a) into (34a) gives the components $E_{x}^{r}(x, z)$ and $E_{z}^{r}(x, z)$ and a similar result is obtained for $\xi>-\omega / c$.

The comparison of (34) and (38a) shows that $H_{y}^{r}(x, z)$ has formally the same expression for $|\xi| \leq \omega / c$ and $|\xi|>\omega / c$, the difference intervening in the coefficients $a_{1}\left(k_{0} / n_{1}\right)$ and $a_{1}^{\dagger}\left(k_{0} / n_{1}\right)$ respectively defined according to (38) and (39a) with a tangent and an hyperbolic tangent

$$
a_{1}=\varepsilon_{0} / \varepsilon_{1} \lambda_{1} \tan \left[\lambda_{1} d\right], a_{1}^{\dagger}=\varepsilon_{1} / \varepsilon_{1} \lambda_{1}^{\dagger} \tanh \left[\lambda_{1}^{\dagger} d\right]
$$

with $a, \lambda$ written for $a\left(k_{0} / n_{1}\right)$ and $\lambda\left(k_{0} / n_{1}\right)$. Then, according to (35) and (39) the reflection coefficients for $|\xi| \leq \omega / c$ and $|\xi|>\omega / c$ are

$$
\begin{gathered}
R=\left(i k_{0} \cos \theta-a_{1}\right)\left(i k_{0} \cos \theta+a_{1}\right)^{-1}, \\
R^{\dagger}=\left(i k_{0} \cos \theta-a_{1}^{\dagger}\right)\left(i k_{0} \cos \theta+a_{1}^{\dagger}\right)^{-1} .
\end{gathered}
$$

These reflection coefficients have a modulus unity $|R|=$ $\left|R^{\dagger}\right|=1$ which is important as just discussed, for the reflection of outbursting waves. For a Veselago film with a periodic thickness, the differences between the phases of $R$ and $R^{\dagger}$ would appear more clearly.

\section{DISCUSSION}

The $H_{y}{ }^{i}$ component of the incident TM field is solution of the 2D-Helmholtz equation

$$
\left(\partial_{x}^{2}+\partial_{z}^{2}+\omega^{2} n_{0}^{2} / c^{2}\right) H_{y}^{i}(x, z)=0
$$

but the general solution of Eq.(42), needed to describe an arbitrary TM electromagnetic beam can be put in the form of an angular spectrum of plane waves $[7,8]$

$$
H_{y}{ }^{i}(x, z)=\int C d \theta F_{y}(\theta) \exp \left[i \omega n_{0} / c(x \sin \theta+z \cos \theta)\right]
$$

in which $F_{y}(\theta)$ is a form factor and $C$ a fixed path of integration in the complex $\theta$-plane so that this spectrum is made of harmonic and evanescent waves and since, as previously discussed, these evanescent waves do not propagate the information they convey is lost and it was proved how in Veselago films, this information can be recovered, evanescent waves being transformed into outbursting waves. Because wave propagation through lenses may be described by an integral of the type (43), the lost information results in a limit of the image resolution and lens performances could be improved by a judicious use of Veselago materials [9], even if some doubts have arisen on the possibility to manufacture such lenses [10].

As mentioned in the introduction, dielectric thin films in many optical applications $[11,12]$ are used as filters with this term understood in its widest sense, including antireflection coatings, mirrors, edge filters, beam splitters and narrow band filters. Hard works supported by many industrial companies have been realized concerning in particular the coating quality [13] to improve their performance and research is booming in such domains as microelectronics and photonics applications [13-15]. Since as just explained, Veselago films do not support information loss, we can dream of "perfect" filters performing exactly their theoretical objective: shorts wave pass, long wave pass, band pass, notch pass and realized by a judicious architectural combination of dielectric and Veselago materials.

We have only considered in this work TM electromagnetic waves because its objective was to compare the scattering properties of dielectric and Veselago films. Clearly similar results, at the expense of more calculations, would be obtained for TE and arbitrary electromagnetic plane waves. 
[3] J.B. Pendry, A.J. Holden, D.J. Robbins, and W.J. Stewart, Trans. Microwave Theory Tech. 47, 2075 (1999).

[4] R.A. Shelbi, D.R. Smith, and S. Schultz, Science 292, 77 (2001).

[5] J.B. Pendry and D.R. Smith, Physics Today 57, 37 (2004); Science 312, 1780 (2006).

[6] M. Idemen, Essays on the Formal Aspects of Electromagnetic Theory, A. Lakhtakia Ed. (Singapour, World Scientifc, 1993).

[7] E.T. Whittaker and G.N. Watson, A Course of Modern Analysis, (Cambridge, University Press, 1952).

[8] M. Born and E. Wolf, Principles of Optics, (Oxford, Pergamon, 1965)
[9] J.B. Pendry, Phys. Rev. Lett. 85, 3966 (2000); Sience 315, 1226 (2007).

[10] A. Lakhtakia, Int. J. Infrared Mill. Waves 23, 339 (2002).

[11] P.H. Lissberger, Rep. Pro. Phys. 33, 197 (1970).

[12] H.A. MacLeod, Thin Films Optical Filters, (Bristol, IOP Publishing, 2001).

[13] D.A. Buchanan, IBM J. Res. Dev. 43, 23 (1999).

[14] J.B. Pendry, L. Martin-Moreno, and F.J. Garcia-Vidal, Science 305, 847 (2006).

[15] P. Hillion, Electrmgn. Phenomena J. 6, 17 (2006). 